\title{
RISK FACTORS FOR FEMALE INFERTILITY AT A TERTIARY HEALTH FACILITY IN AKURE, SOUTH-WEST NIGERIA
}

Background. The inability of couples to achieve pregnancy is a major cause of psycho-social problems in family relationship that could lead to marital disharmony.

Objective. The aim of this study was to find out the possible risk factors for female infertility.

Methods. A case-control design and a sample size of 400 (200 cases of infertility and 200 controls) were used in the study. Cases and controls were selected at random at the infertility and family planning clinic of the University of Medical Sciences Teaching Hospital Complex, Akure and were subjected to a predesigned interviewer administered questionnaire to collect the data. The cases were classified into primary and secondary infertility; binary and stepwise logistic regressions were used to generate the Odds ratio and 95\% confidence interval of the possible risk factors and the level of significance was set at $P<0.05$.

Results. The mean age of the women with infertility was $28.5 \pm 5.43$ years and the mean age of those in the control group was $29.1 \pm 5.62$ years. Among the cases, 155 (77.5\%) had secondary infertility, while 45 (22.5\%) had primary infertility. Significant risk factors for female infertility included presence of fibroids, having had fibroid operation, multiple sexual partners, previous abortion, polycystic ovary syndrome (PCOS), sexually transmitted infection (STI) and post abortion sepsis.

Conclusion. The study showed that secondary infertility is still the most prevalent and the risk factors were multi factorial. Efforts should be intensified to reduce infertility due to preventable causes.

KEY WORDS: female infertility; risk factors; fibroids; polycystic ovary syndrome; sexually transmitted infection.

\section{Introduction}

Infertility is the inability of a couple to achieve pregnancy over an average period of one year despite adequate, regular unprotected sexual intercourse [1]. WHO in 1991 estimated that between 8 and $12 \%$ of couples experienced some form of infertility during their reproductive lives, thus affecting 50 to 80 million worldwide, out of which 20-35 million couples in Africa expected to experience this problem [2]. This can be extrapolated to 3-4 million Nigeria couples suffering from infertility [3]. According to Ogunniyi in 1995, the prevalence of infertility in Sub-Saharan Africa was reported as ranging between $20-60 \%$ [4]. However, the estimate of infertile couples in Ile-Ife has been put at 19\% by Okonofua in 1995 [5], although authors in previous studies in the other parts of Nigeria presented different ranging estimates.

Corresponding Author: Dr. Theresa Azonima Irinyenikan, Consultant Obstetrician and Gynaecologist, Department of Obstetrics and Gynaecology, Faculty of Clinical Sciences, University of Medical Sciences/University of Medical Sciences Teaching Hospital Complex, Akure, Ondo State, Nigeria.

E-mail: tirinyenikan2017@gmail.com
Causes of infertility could be due to female factors, male factors or both. Estimates show that in $35-40 \%$ of cases a man is infertile and in $35-40 \%$ of cases a woman is infertile while in $20-30 \%$ of cases, it is related to the combination of other factors [6]. Causes of female infertility include conditions that may damage the fallopian tubes, interfere with ovulation or cause hormonal imbalance [7]. These conditions include pelvic inflammatory disease resulting from sexually transmitted infections, endometriosis, polycystic ovarian syndrome, premature ovarian failure, uterine fibroids and environmental factors [7]. Other causes of infertility in females include post abortion sepsis, puerperal sepsis and age-related factors [8]. The risk factors for infertility can therefore be classified into: genital, endocrinal, developmental and general factors [9].

Infertility can be primary, if a woman has never conceived before, or it can be secondary, if a woman has at least once conceived but may or may not have carried the pregnancy to term [10]. In resource-rich countries primary infer- 
tility is much more common than secondary infertility, however the reverse is the case in Sub-Saharan Africa (SSA) [11]. In some African regions, the prevalence of secondary infertility is more than $30 \%$ [12]. The high secondary infertility rate in SSA is thought to be due to sexually transmitted infections (STIs) and medical interventions under unhygienic conditions, particularly during delivery or induced abortions [13].

Infertility is a global problem affecting people around the world, which cause and importance may vary according to the geographical location and socio-economic condition; assessing risk factors for female infertility should be geographical considering the variations that could arise from different regions. The aim of this study was to evaluate the risk factors for female infertility among cases of infertility presenting to a tertiary facility in Akure, South-West Nigeria, and the type of infertility prevalent among women there. This will further help to highlight the burden of this condition and subsequently help in policy making.

\section{Methods}

\section{Study design}

The study used a matched case control design and was conducted at the infertility clinic and family planning clinic of the University of Medical Sciences Teaching Hospital Complex, Akure over a period of 2 years (July 2017- June 2019).

$$
\begin{aligned}
& \text { Sample size calculation } \\
& \qquad n=\left(\frac{r+1}{r}\right) \frac{(\bar{p})(1-\bar{p})\left(Z_{\beta}+Z_{\alpha 2}\right)^{2}}{\left(p_{1}-p_{2}\right)^{2}}
\end{aligned}
$$

where $n$ - proportion in each group; $p_{2}$ proportion exposed in the control group $=20 \%$; $p_{1}$ - proportion of cases exposed; $\bar{p}$ - average proportion of cases exposed; $r=1$ (equal number of cases and controls), using $80 \%$ power, $Z_{\beta}=0.84$ and for $5 \%$ significance level using an odd ratio of $2.0, Z_{\alpha}=1.96$

$$
\begin{aligned}
& p_{1}=2.0(.20) /(.20)(2.0-1)+1=0.40 / 1.20=0.33, \\
& \bar{p}=(.33+.20)=0.265 \\
& n=2 \frac{(.256)(1-.265)(.84+1.96)^{2}}{(.33-.20)^{2}}=181
\end{aligned}
$$

Sample size $=2 \times 181=362$, this was rounded up to 400 .

A total of 200 cases and an age matched 200 controls enrolled into the study.

Consent: Informed consent was obtained from all the clients who participated in this study.

Ethical Approval: The ethical approval for the study was obtained from the Ethics Com- mittee of the University of Medical Sciences Teaching Hospital Complex Akure, Ondo State. Protocol number 015, 12 $2^{\text {th }}$ May 2017.

Inclusion criteria:Cases were infertile women who have attended the infertility clinic at least twice and the controls were age matched women attending the family planning clinic of the same hospital that gave their consent to participate in the study.

Exclusion criteria: Women who had medications or surgery to induce pregnancy among the controls and those who did not give consent.

\section{Sampling technique}

Cases (infertile women) were selected at random from the infertility clinic of the hospital through a weekly visit till the required sample size was obtained. Controls involved fertile women, who attended the family planning clinic of the same hospital. The cases and controls were subjected to a predesigned interviewer administered questionnaire to collect sociodemographic data such as age, occupation, educational level, age at marriage; menstrual history such as age of menarche, regularity of menses, family history of infertility; relevant medical history such as diabetes mellitus, thyroid diseases, hypertension were also assessed; surgical history such as abdominal/pelvic surgeries were obtained. Information on probable gynaecological conditions that could cause infertility such as history of endometriosis, polycystic ovarian syndrome, presence of fibroids, sexually transmitted infections, genital infection following childbirth and previous abortion were obtained. The cases were classified into primary or secondary infertility.

\section{Statistical Analysis}

The collected data were analysed using the computer package SPSS version 23. Descriptive tables were generated and binary logistic regression was used to generate the Odds ratio and the $95 \%$ confidence interval of the possible risk factors for infertility. These factors were further subjected to analysis using a stepwise logistic regression to identify the main predictors of female infertility. The statistical significance was set at $p<0.05$.

\section{Results}

During the study, 200 cases of female infertility were registered and 200 age-matched controls. The mean age of the women with infertility was $28.5 \pm 5.43$ years old and the mean age of those in the control group was $29.1 \pm 5.62$ years old. Among the cases, 155 (77.5\%) pa- 
tients suffered from secondary infertility, while $45(22.5 \%)$ had primary infertility. The mean ages of those with secondary and primary infertility were $28.7 \pm 4.4$ years old and $29.1 \pm 3.4$ years old respectively. As presented in Table 1 , $96(61.9 \%)$ cases of secondary infertility and 70 $(45.2 \%)$ of their control were traders, the risk of secondary infertility was statistically significantly higher among the traders compare to their controls $[\mathrm{OR}=0.17,95 \% \mathrm{CI}=0.06-0.48$, $\mathrm{p}=0.001]$. Meanwhile, there was no statistically significant difference between the occupation of those with primary infertility and their control. The majority of the subjects practiced Christian religion, were married and had tertiary level of education with no statistically significant difference ( $p>0.05)$ in both groups.

Table 2 showed the distribution of female infertility and the controls according to their gynaecological history.

Most of the women in both groups were already married at the age of over 18 years old. Among those with secondary infertility, 94 (60.6\%) had no living children but had abortions/ miscarriages in the past compare to their controls, where 92 (59.4\%) already had $\geq 3$ children, this was statistically significant, $p=0.000$. Similarly, the $45(100 \%)$ of those with primary infertility have never been pregnant compare to their controls, where 31 (68.9\%) had $\geq 3$ living children. This was also statistically significant, $p=0.000$. Among those with secondary infertility, $31(20.0 \%)$ had more than one sexual partner compare to $4(2.6 \%)$ of the control, while 7 $(15.6 \%)$ of those with primary infertility and 0 $(0 \%)$ of the control had more than one sexual partner; this was also statistically significant, $\mathrm{P}=0.000$. Among the cases, 91 (58.7\%) of those with secondary infertility started their menses after the age of 15 years compare to 88 (56.8\%) of their control, delayed menses was not a significant risk factor for infertility [OR $=0.92$, $\mathrm{CI}=0.59-1.45, \mathrm{p}=0.730]$. Only $10(6.5 \%)$ of the cases of secondary infertility and $3(6.7 \%)$ of their control reported family history of infertility, this was not a significant risk factor for infertility [OR=0.89, CI=0.35-2.26, p=0.813].

The analysis of cases of female infertility and the control according to the medical or surgical history showed that none of the subjects have ever had diabetes, thyroid disease or tuberculosis. Though, $10(6.5 \%)$ of the cases of secondary infertility and 7 (4.5\%) of their control reported to have had hypertension while $3(6.7 \%)$ of the cases of primary infertility and none of their control reported to

Table 1. Socio-demographic characteristics of cases of female infertility and control subjects

\begin{tabular}{|c|c|c|c|c|c|c|c|c|}
\hline $\begin{array}{l}\text { Demographic } \\
\text { factors }\end{array}$ & $\begin{array}{c}2^{\circ} \text { infertility } \\
\text { cases } \\
(n=155) \\
\text { No }(\%)\end{array}$ & $\begin{array}{l}\text { Control } \\
(n=155) \\
\text { No }(\%)\end{array}$ & $\begin{array}{c}\text { OR } \\
(95 \% \mathrm{CI})\end{array}$ & $\begin{array}{c}\mathrm{P} \\
\text { value }\end{array}$ & $\begin{array}{c}1^{\circ} \text { infertility } \\
\text { cases } \\
(n=45) \\
\text { No }(\%)\end{array}$ & $\begin{array}{l}\text { Control } \\
(n=45) \\
\text { No }(\%)\end{array}$ & $\begin{array}{c}\text { OR } \\
(95 \% \mathrm{CI})\end{array}$ & $\begin{array}{c}\mathrm{P} \\
\text { value }\end{array}$ \\
\hline $\begin{array}{l}\text { Age } \\
15-24 \\
25-34 \\
\geq 35 \\
\end{array}$ & $\begin{array}{c}0(0.0) \\
78(50.3) \\
77(49.7)\end{array}$ & $\begin{array}{c}3(1.9) \\
73(47.1) \\
79(51.0) \\
\end{array}$ & - & 0.203 & $\begin{array}{r}2(4 \\
26(5 \\
17(3 \\
\end{array}$ & $\begin{array}{r}1(2 \\
19(4 \\
25(5\end{array}$ & $\begin{array}{c}0.34 \\
(0.06-1.96) \\
0.49 \\
(0.27-0.91) \\
\end{array}$ & 0.229 \\
\hline $\begin{array}{l}\text { Occupation } \\
\text { Civil/public } \\
\text { servants } \\
\text { Trading/ } \\
\text { business } \\
\text { Housewives/ } \\
\text { applicants } ® \\
\end{array}$ & $\begin{array}{c}54(34.8) \\
96(61.9) \\
5(3.2)\end{array}$ & $\begin{array}{l}64(41.3) \\
70(45.2) \\
21(13.5)\end{array}$ & $\begin{array}{c}0.28 \\
(0.10-0.79) \\
0.17 \\
(0.06-0.48)\end{array}$ & 0.001 & $\begin{array}{c}15(33.3) \\
26(57.8) \\
4(8.9)\end{array}$ & $\begin{array}{c}22(48.9) \\
22(48.9) \\
1(2.2)\end{array}$ & $\begin{array}{c}5.86 \\
(0.59-57.78) \\
3.38 \\
(0.35-32.55)\end{array}$ & 0.177 \\
\hline $\begin{array}{l}\text { Religion } \\
\text { Christian } \\
\text { Islam } \\
\end{array}$ & $\begin{array}{c}148(95.5) \\
7(4.5) \\
\end{array}$ & $\begin{array}{r}147 \\
8( \\
\end{array}$ & $\begin{array}{c}0.86 \\
(0.31-2.45) \\
\end{array}$ & 0.791 & $\begin{array}{c}44(97.8) \\
1(2.2) \\
\end{array}$ & $\begin{array}{c}42(93.3) \\
3(6.7) \\
\end{array}$ & $\begin{array}{c}0.32 \\
(0.06-1.62) \\
\end{array}$ & 0.306 \\
\hline $\begin{array}{l}\text { Marital status } \\
\text { Single } \\
\text { Married }\end{array}$ & $\begin{array}{c}3(1.9) \\
152(98.1)\end{array}$ & $\begin{array}{c}1(0.6) \\
154(99.4)\end{array}$ & $\begin{array}{c}0.33 \\
(0.03-3.19) \\
\end{array}$ & 0.314 & $\begin{array}{c}0(0.0) \\
45(100)\end{array}$ & $\begin{array}{c}2(4.4) \\
43(95.6)\end{array}$ & - & 0.153 \\
\hline $\begin{array}{l}\text { Educational } \\
\text { level } \\
\text { Primary } \\
\text { Secondary } \\
\text { Tertiary }{ }^{\circledR}\end{array}$ & $\begin{array}{c}13(8.4) \\
46(29.7) \\
96(61.9)\end{array}$ & $\begin{array}{c}7(4.5) \\
38(24.5) \\
110(71.0)\end{array}$ & $\begin{array}{c}0.47 \\
(0.18-1.23) \\
0.72 \\
(0.43-1.20) \\
\text { Reference } \\
\end{array}$ & 0.173 & $\begin{array}{c}3(6.7) \\
12(26.7) \\
30(66.7)\end{array}$ & $\begin{array}{c}2(4.4) \\
13(28.9) \\
30(66.7)\end{array}$ & $\begin{array}{c}0.67 \\
(1.10-4.28) \\
1.08 \\
(0.42-2.75) \\
\text { Reference }\end{array}$ & 0.887 \\
\hline
\end{tabular}

$\circledR=$ reference category 
Table 2. Distribution of cases of female infertility and controls according to their gynaecological history

\begin{tabular}{|c|c|c|c|c|c|c|c|c|}
\hline Factors & $\begin{array}{c}2^{\circ} \text { infertility } \\
\text { cases } \\
(n=155) \\
\text { No }(\%)\end{array}$ & $\begin{array}{l}\text { Control } \\
(n=155) \\
\text { No }(\%)\end{array}$ & $\begin{array}{c}\text { OR } \\
(95 \% \mathrm{CI})\end{array}$ & $\begin{array}{c}P \\
\text { value }\end{array}$ & $\begin{array}{c}1^{\circ} \text { infertility } \\
\text { cases } \\
(n=45) \\
\text { No }(\%)\end{array}$ & $\begin{array}{l}\text { Control } \\
(n=45) \\
\text { No }(\%)\end{array}$ & $\begin{array}{c}\text { OR } \\
(95 \% \mathrm{CI})\end{array}$ & $\begin{array}{c}P \\
\text { value }\end{array}$ \\
\hline $\begin{array}{l}\text { Age at marriage } \\
<18 \\
\geq 18 \\
\text { None }\end{array}$ & $\begin{array}{c}2(1.3) \\
151(97.4) \\
2(1.3) \\
\end{array}$ & $\begin{array}{c}0(0.0) \\
155(100) \\
0(0.0) \\
\end{array}$ & - & 0.132 & $\begin{array}{c}0(0.0) \\
45(100) \\
0(0.0)\end{array}$ & $\begin{array}{c}1(2.2) \\
43(95.6) \\
1(2.2) \\
\end{array}$ & - & 0.360 \\
\hline $\begin{array}{l}\text { Parity } \\
1 \\
2 \\
\geq 3 \\
\text { None }\end{array}$ & $\begin{array}{c}46(29.7) \\
13(8.4) \\
2(1.3) \\
94(60.6) \\
\end{array}$ & $\begin{array}{c}20(12.9) \\
43(27.7) \\
92(59.4) \\
0(0.0) \\
\end{array}$ & - & 0.000 & $\begin{array}{c}0(0.0) \\
0(0.0) \\
0(0.0) \\
45(100)\end{array}$ & $\begin{array}{c}2(4.4) \\
12(26.7) \\
31(68.9) \\
0(0.0) \\
\end{array}$ & - & 0.000 \\
\hline $\begin{array}{l}\text { Age at } 1^{\text {st }} \\
\text { menses } \\
<15 \AA \\
\geq 15\end{array}$ & $\begin{array}{l}64(41.3) \\
91(58.7) \\
\end{array}$ & $\begin{array}{l}67(43.2) \\
88(56.8)\end{array}$ & $\begin{array}{c}0.92 \\
(0.59-1.45)\end{array}$ & 0.730 & $\begin{array}{l}23(51.1) \\
22(48.9) \\
\end{array}$ & $\begin{array}{l}17(37.8) \\
28(62.2) \\
\end{array}$ & $\begin{array}{c}0.58 \\
(0.32-1.05)\end{array}$ & 0.203 \\
\hline $\begin{array}{l}\text { Family history } \\
\text { of infertility } \\
\text { Yes } \\
\text { No } \\
\end{array}$ & $\begin{array}{c}10(6.5) \\
145(93.5)\end{array}$ & $\begin{array}{c}9(5.8) \\
146(94.2) \\
\end{array}$ & $\begin{array}{c}0.89 \\
(0.35-2.26)\end{array}$ & 0.813 & $\begin{array}{c}3(6.7) \\
42(93.3)\end{array}$ & $\begin{array}{c}0(0.0) \\
45(100)\end{array}$ & - & 0.078 \\
\hline $\begin{array}{l}\text { No sexual } \\
\text { partner } \\
1 \\
>1\end{array}$ & $\begin{array}{c}124(80.0) \\
31(20.0)\end{array}$ & $\begin{array}{c}151(97.4) \\
4(2.6)\end{array}$ & $\begin{array}{c}9.43 \\
(3.24-27.46)\end{array}$ & 0.000 & $\begin{array}{c}38(84.4) \\
7(15.6)\end{array}$ & $\begin{array}{c}45(100.0) \\
0(0.0)\end{array}$ & - & 0.006 \\
\hline
\end{tabular}

have had hypertension, this was not statistically control [OR=0.06, 95\% CI=0.01-0.47, $\mathrm{P}=0.007$ ]. significant $[\mathrm{OR}=0.69, \mathrm{CI}=0.25-1.85, \mathrm{P}=0.454]$. Also, $5(11.1 \%)$ of those with primary infertility However, $15(9.7 \%)$ of secondary infertility cases and none of the control have had fibroid have had fibroid operation compare with 1 operation, the risk of primary infertility was $(0.6 \%)$ of the control and the risk of secondary significantly higher than the control, $\mathrm{P}=0.021$ infertility was significantly higher than the (Table 3).

Table 3. Distribution of cases of female infertility with controls according to their medical/surgical history

\begin{tabular}{|c|c|c|c|c|c|c|c|c|}
\hline $\begin{array}{c}\text { Medical } \\
\text { conditions }\end{array}$ & $\begin{array}{c}2 \circ \text { infertility } \\
\text { cases } \\
(n=155) \\
\text { No }(\%)\end{array}$ & $\begin{array}{l}\text { Control } \\
(n=155) \\
\text { No }(\%)\end{array}$ & $\begin{array}{c}\text { OR } \\
(95 \% \mathrm{CI})\end{array}$ & $\begin{array}{c}P \\
\text { value }\end{array}$ & $\begin{array}{c}10 \text { infertility } \\
\text { cases } \\
(n=45) \\
\text { No }(\%)\end{array}$ & $\begin{array}{l}\text { Control } \\
(n=45) \\
\text { No }(\%)\end{array}$ & $\begin{array}{c}\text { OR } \\
(95 \% \mathrm{CI})\end{array}$ & $\begin{array}{c}P \\
\text { value }\end{array}$ \\
\hline $\begin{array}{l}\text { Diabetes } \\
\text { Yes } \\
\text { No }\end{array}$ & $\begin{array}{c}0(0.0) \\
155(100)\end{array}$ & $\begin{array}{c}0(0.0) \\
155(100)\end{array}$ & - & - & $\begin{array}{c}0(0.0) \\
45(100)\end{array}$ & $\begin{array}{c}0(0.0) \\
45(100)\end{array}$ & - & - \\
\hline $\begin{array}{l}\text { Hypertension } \\
\text { Yes } \\
\text { No }\end{array}$ & $\begin{array}{c}10(6.5) \\
145(93.5)\end{array}$ & $\begin{array}{c}7(4.5) \\
148(95.5)\end{array}$ & $\begin{array}{c}0.69 \\
(0.25-1.85)\end{array}$ & 0.454 & $\begin{array}{c}3(6.7) \\
42(93.3)\end{array}$ & $\begin{array}{c}0(0.0) \\
45(100)\end{array}$ & - & 0.078 \\
\hline $\begin{array}{l}\text { Thyroid } \\
\text { Yes } \\
\text { No }\end{array}$ & $\begin{array}{c}0(0.0) \\
155(100)\end{array}$ & $\begin{array}{c}0(0.0) \\
155(100)\end{array}$ & - & - & $\begin{array}{c}0(0.0) \\
45(100)\end{array}$ & $\begin{array}{c}0(0.0) \\
45(100)\end{array}$ & - & - \\
\hline $\begin{array}{l}\text { Tuberculosis } \\
\text { Yes } \\
\text { No }\end{array}$ & $\begin{array}{c}- \\
155(100)\end{array}$ & $\begin{array}{c}- \\
155(100)\end{array}$ & - & - & $\begin{array}{c}- \\
45(100)\end{array}$ & $\begin{array}{c}- \\
45(100)\end{array}$ & - & - \\
\hline $\begin{array}{l}\text { Fibroid operation } \\
\text { Yes } \\
\text { No }\end{array}$ & $\begin{array}{c}15(9.7) \\
140(90.3)\end{array}$ & $\begin{array}{c}1(0.6) \\
154(99.4)\end{array}$ & $\begin{array}{c}0.06 \\
(0.01-0.47)\end{array}$ & 0.000 & $\begin{array}{c}5(11.1) \\
40(88.9) \\
\end{array}$ & $\begin{array}{c}0(0.0) \\
45(100)\end{array}$ & - & 0.021 \\
\hline $\begin{array}{l}\text { Other operation } \\
\text { Yes } \\
\text { No }\end{array}$ & $\begin{array}{c}1(0.6) \\
154(99.4)\end{array}$ & $\begin{array}{c}0(0.0) \\
155(100)\end{array}$ & - & 0.317 & $\begin{array}{c}0(0.0) \\
45(100)\end{array}$ & $\begin{array}{c}0(0.0) \\
45(100)\end{array}$ & - & - \\
\hline
\end{tabular}


Table 4 presents the analysis of cases of infertility and the control according to their gynaecological conditions. The gynaecological conditions in the subjects included fibroids, which was present in $46(29.7 \%)$ cases of secondary infertility compare to $11(7.1 \%)$ of their control, the risk of secondary infertility among them was significantly higher than their control [OR=0.18, 95\% CI=0.09-0.37, P=0.00].

Similarly, among the cases of primary infertility, 15 (33.3\%) had fibroids compare to only $1(2.2 \%)$ of their control, the risk of primary infertility was significantly higher than the control [OR=0.07, 95\%CI=0.008-0.64, $\mathrm{P}=0.00]$. Also, among the cases of secondary infertility, $50(32.3 \%)$ had endometriosis, $35(22.6 \%)$ of their control had similar condition, while in the cases of primary infertility 15 (33.3\%) had endometriosis and 10 (22.2\%) had the condition, there was no statistically significant difference in both groups at $p>0.05$. Among the 2 groups, $114(73.5 \%)$ of those with secondary infertility had previous abortion compare to 53 (34.2\%) of their control while none was reported in the cases of primary infertility. The risk of secondary infertility was significantly higher than the control [OR=0.19, 95\% CI=0.12-0.30, $\mathrm{p}=0.000]$. Post abortion sepsis was reported in 30 (19.4\%) of cases of secondary infertility and only $10(6.5 \%)$ of the control with a statistically significant higher risk among the cases [OR $=0.29$, $95 \% \mathrm{CI}=0.14-0.61, \mathrm{p}=0.001]$. Another gynaecological condition prevalent was polycystic ovary syndrome (PCOS), which was reported in $53(34.2 \%)$ cases of secondary infertility and $7(4.5 \%)$ of their control, the risk of PCOS among those with secondary infertility was significantly higher than their control, [OR=0.09, 95\% $\mathrm{CI}=0.04-0.21, \mathrm{p}=0.000]$ while in the cases of primary infertility, it was reported in 18 (40.0\%) and $2(4.4 \%)$ of their control and the risk of PCOS was also significantly higher than the control [OR=0.01, 95\% CI=0.01-0.52, $p=0.000]$. Sexually transmitted infection was reported in $47(30.3 \%)$ cases of secondary infertility and 18 $(11.6 \%)$ of their control with a significantly higher risk of this condition among these cases [OR=0.30,95\% CI=0.17-0.55, $\mathrm{p}=0.000$ ), while

Table 4. Analysis of cases of female infertility and the controls according to their gynaecological conditions

\begin{tabular}{|c|c|c|c|c|c|c|c|c|}
\hline $\begin{array}{l}\text { Gynaecological } \\
\text { conditions }\end{array}$ & $\begin{array}{c}2 \circ \text { infertility } \\
\text { cases } \\
(n=155) \\
\text { No }(\%)\end{array}$ & $\begin{array}{l}\text { Control } \\
(n=155) \\
\text { No }(\%)\end{array}$ & $\begin{array}{c}\text { OR } \\
(95 \% \mathrm{CI})\end{array}$ & $\begin{array}{c}\mathrm{P} \\
\text { value }\end{array}$ & $\begin{array}{c}10 \text { infertility } \\
\text { cases } \\
(n=45) \\
\text { No }(\%)\end{array}$ & $\begin{array}{l}\text { Control } \\
(n=45) \\
\text { No }(\%)\end{array}$ & $\begin{array}{c}\text { OR } \\
(95 \% \mathrm{CI})\end{array}$ & $\begin{array}{c}\mathrm{P} \\
\text { value }\end{array}$ \\
\hline $\begin{array}{l}\text { Endometriosis } \\
\text { Yes } \\
\text { No }\end{array}$ & $\begin{array}{c}50(32.3) \\
105(67.7)\end{array}$ & $\begin{array}{c}35(22.6) \\
120(77.4)\end{array}$ & $\begin{array}{c}0.61 \\
(0.37-1.02) \\
\end{array}$ & 0.056 & $\begin{array}{l}15(33.3) \\
30(66.7)\end{array}$ & $\begin{array}{l}10(22.2) \\
35(77.8)\end{array}$ & $\begin{array}{c}0.79 \\
(0.17-3.69)\end{array}$ & 0.239 \\
\hline $\begin{array}{l}\text { Fibroid } \\
\text { diagnosis } \\
\text { Yes } \\
\text { No }\end{array}$ & $\begin{array}{c}46(29.7) \\
109(70.3)\end{array}$ & $\begin{array}{c}11(7.1) \\
144(92.9)\end{array}$ & $\begin{array}{c}0.18 \\
(0.09-0.37)\end{array}$ & 0.000 & $\begin{array}{l}15(33.3) \\
30(66.7)\end{array}$ & $\begin{array}{c}1(2.2) \\
44(97.8)\end{array}$ & $\begin{array}{c}0.07 \\
(0.008-0.64)\end{array}$ & 0.000 \\
\hline $\begin{array}{l}\text { Previous } \\
\text { abortion } \\
\text { Yes } \\
\text { No }\end{array}$ & $\begin{array}{c}114(73.5) \\
41(26.5)\end{array}$ & $\begin{array}{c}53(34.2) \\
102(65.8)\end{array}$ & $\begin{array}{c}0.19 \\
(0.12-0.30)\end{array}$ & 0.000 & $\begin{array}{c}0(0.0) \\
45(100)\end{array}$ & $\begin{array}{l}16(35.6) \\
29(64.4)\end{array}$ & - & 0.000 \\
\hline $\begin{array}{l}\text { PCOS } \\
\text { Yes } \\
\text { No }\end{array}$ & $\begin{array}{c}53(34.2) \\
102(65.8)\end{array}$ & $\begin{array}{c}7(4.5) \\
148(95.5)\end{array}$ & $\begin{array}{c}0.09 \\
(0.04-0.21)\end{array}$ & 0.000 & $\begin{array}{l}18(40.0) \\
27(60.0)\end{array}$ & $\begin{array}{c}2(4.4) \\
43(95.6)\end{array}$ & $\begin{array}{c}0.01 \\
(0.01-0.52) \\
\end{array}$ & 0.000 \\
\hline $\begin{array}{l}\text { Menstrual cycle } \\
\text { Regular } \\
\text { Irregular }\end{array}$ & $\begin{array}{c}141(91.0) \\
14(9.0)\end{array}$ & $\begin{array}{c}147(94.8) \\
8(5.2)\end{array}$ & $\begin{array}{c}0.55 \\
(0.22-1.35)\end{array}$ & 0.184 & $\begin{array}{c}37(82.2) \\
8(17.8)\end{array}$ & $\begin{array}{c}41(91.1) \\
4(8.9)\end{array}$ & $\begin{array}{c}0.83 \\
(0.10-6.71)\end{array}$ & 0.215 \\
\hline $\begin{array}{l}\text { Genital infection } \\
\text { Yes } \\
\text { No }\end{array}$ & $\begin{array}{c}8(5.2) \\
147(94.8)\end{array}$ & $\begin{array}{c}10(6.5) \\
145(93.5)\end{array}$ & $\begin{array}{c}1.26 \\
(0.48-3.30)\end{array}$ & 0.627 & $\begin{array}{c}0(0.0) \\
45(94.8)\end{array}$ & $\begin{array}{c}4(8.9) \\
41(91.1)\end{array}$ & - & 0.061 \\
\hline $\begin{array}{l}\text { STI } \\
\text { Yes } \\
\text { No }\end{array}$ & $\begin{array}{c}47(30.3) \\
108(69.7)\end{array}$ & $\begin{array}{c}18(11.6) \\
137(88.4)\end{array}$ & $\begin{array}{c}0.30 \\
(0.17-0.55)\end{array}$ & 0.000 & $\begin{array}{c}6(13.3) \\
39(86.7)\end{array}$ & $\begin{array}{c}7(15.6) \\
38(84.4)\end{array}$ & $\begin{array}{c}1.11 \\
(0.21-5.89)\end{array}$ & 0.764 \\
\hline $\begin{array}{l}\text { Post abortion } \\
\text { sepsis } \\
\text { Yes } \\
\text { No }\end{array}$ & $\begin{array}{c}30(19.4) \\
125(80.6)\end{array}$ & $\begin{array}{c}10(6.5) \\
145(93.5)\end{array}$ & $\begin{array}{c}0.29 \\
(0.14-0.61)\end{array}$ & 0.001 & $\begin{array}{c}0(0.0) \\
45(100)\end{array}$ & $\begin{array}{c}3(6.7) \\
42(93.3)\end{array}$ & $\begin{array}{c}0.71 \\
(0.0-0.0)\end{array}$ & 0.078 \\
\hline
\end{tabular}


among the cases of primary infertility 6 (13.3\%) and $7(15.6 \%)$ of their control reported to have had this condition, this was not statistically significant, $p>0.05$. Among the cases of secondary infertility, 8 (5.2\%) and $106.5 \%)$ of the control had genital infection, there was no statistically significant difference in both groups. Menstrual irregularity was found in 14 (9.0\%) cases of infertility compare to 8 (5.2\%) of their control and $8(17.8 \%)$ of primary infertility compare to $4(8.9 \%)$ of the control, this was not statistically significant.

Table 5 showed various predictors of secondary and primary infertility using stepwise logistic regression analysis of the various independent risk factors for infertility.

Table 5. Stepwise logistic regression to identify predictors of female infertility

\begin{tabular}{|l|c|c|c|c|c|c|}
\hline \multicolumn{1}{|c|}{ Independent variable } & B & $\begin{array}{c}\text { S.E. } \\
\text { (Standard } \\
\text { Error) }\end{array}$ & Test & P. value & OR & 95\% CI \\
\hline Model (1): For Secondary infertility & & & & & & \\
\hline Fibroid diagnosis & 1.113 & 0.426 & 6.838 & 0.009 & 3.04 & $1.32-7.01$ \\
\hline Fibroid operation & 1.621 & 1.151 & 1.984 & 0.159 & 5.06 & $0.53-48.21$ \\
\hline PCOS & 2.213 & 0.45 & 24.174 & 0.00 & 9.14 & $3.78-22.08$ \\
\hline STI & 0.746 & 0.359 & 4.325 & 0.038 & 2.11 & $1.04-4.26$ \\
\hline Post abortion sepsis & -0.271 & 0.473 & 0.329 & 0.57 & 0.76 & $0.30-1.93$ \\
\hline Previous abortion & 1.544 & 0.294 & 27.564 & 0.000 & 4.68 & $2.63-8.33$ \\
\hline Constant & -12.393 & 2.50 & 24.487 & & & \\
\hline Model (2):For Primary infertility & & & & & & \\
\hline Fibroid diagnosis & 2.508 & 1.118 & 5.034 & 0.025 & 12.28 & $1.37-109.86$ \\
\hline PCOS & 2.884 & 1.13 & 6.489 & 0.01 & 17.88 & $1.94-164.51$ \\
\hline STI & -0.052 & 0.84 & 0.004 & 0.951 & 0.949 & $0.18-4.94$ \\
\hline Constant & -12.393 & 2.50 & 24.487 & 0.000 & & \\
\hline
\end{tabular}

In Model 1, among the various significant factors detected by the binary logistic regression analysis for secondary infertility only the presence of fibroids [OR=3.04, 95\% CI=1.32-7.01, $\mathrm{p}=0.009]$, polycystic ovary syndrome [OR $=9.14$, $95 \% \mathrm{CI}=3.78-22.08, \mathrm{P}=0.00]$, sexually transmitted infection [OR=2.11, 95\% CI=1.04-4.26, $\mathrm{p}=0.038$ ] and previous abortion [OR=4.68, 95\% CI $=2.63$ $8.33, p=0.000$ ] were predictors of secondary infertility. In Model 2, the presence of fibroids [OR=12.28, 95\% CI=1.37-109.86, $p=0.025]$ and polycystic ovary syndrome [OR $=17.88,95 \%$ $C I=1.94-164.51, p=0.01]$ were the predictors of primary infertility.

\section{Discussion}

The study showed that secondary infertility was commoner among the women $(77.5 \%)$ than primary infertility (22.5\%), which is in keeping with studies carried out in other parts of Nigeria $[11,12,13]$. It further highlights the burden of secondary infertility which is more prevalent in Sub-Saharan African countries compared to the Western world [14].The demographic characteristics of the subjects showed that most of the women were already married and possibly in a sexual relationship to enable one ascertain if there are issues concerning their fertility.
Other factors such as the woman's age, religion, educational level had no impact on fertility as against a similar study which reported a decrease in a woman's fertility with increasing age [15]. These factors therefore were not risk factors for infertility among our women.

Menstrual irregularity was seen in some of the women with secondary infertility though not statistically significant and therefore suggest that this has no strong effect as a risk factor for infertility among our women. However, having multiple sexual partners was a significant risk factor for infertility among the women, which further confirmed the increase in sexually transmitted infection which was also reported among the women with secondary infertility. Sexually transmitted infections are transmitted through sexual activity with an infected partner and a major cause of secondary infertility in Sub-Saharan Africa [6].

This study also highlights gynaecological conditions such as the presence of fibroids and previous abdominal surgeries for fibroids as risk factors for female infertility which is in keeping with another study [16]. Large fibroids may cause infertility by impairing the uterine lining, blocking the fallopian tube, distorting the shape of the uterine cavity or altering the 
position of the cervix. Also, following pelvic surgery, postsurgical or post infective uterine or abdominal adhesions that result may restrict the movement of ovaries and fallopian tubes and cause infertility.

Previous abortion and post abortion sepsis were significant risk factors for secondary infertility in this study. This finding has also been reported in another study in Nigeria where induced abortion and post abortion sepsis were found to affect future fertility [17]. This may be as a result of repeated injuries to the uterine lining from multiple dilatation and curettage which can cause adhesions within the uterus thus leading to secondary amenorrhea and infertility. However, genital infection did not have any significant effect on the cause of infertility, this could have been due to increase access to health care and the presence of skilled birth attendants during delivery.

This study showed ovarian dysfunction as a result of polycystic ovary syndrome to be a significant cause of both primary and secondary infertility among the women, similar studies have shown that nearly $10 \%$ of infertile women are diagnosed with reduced ovarian dysfunction and polycystic ovary syndrome implicated as a common cause of ovulation disorder in women of childbearing age $[18,19,20]$.

In this study the main predictors of female infertility were the presence of fibroids, polycystic ovary syndrome, sexually transmitted infections and previously induced abortions for unwanted pregnancies. These findings have further revealed the great impact of reproductive infections and hormonal imbalance as major causes of female infertility.

\section{Conclusion}

Female infertility is still a major public health issue and its cause could be multi factorial. Secondary infertility remains the most prevalent type in the region mostly due to tubal damage as a result of increase in sexually transmitted infections and previous induced abortions. Though, hormonal cause such as polycystic ovary syndrome may not be under our control, reproductive infections from sexual activity could be curtailed by preventing unsafe sex and prompt treatment of diseases resulting from sexually transmitted infections. Efforts should be intensified to prevent unsafe abortions which could lead to infertility in the future.

Acknowledgements. I acknowledge the Consultant Obstetricians and Gynaecologists of the University of Medical Sciences Teaching Hospital Complex Akure for allowing to use their patients for the study, the research assistants, who helped in the collection of the data and the statistician, who analysed the data.

\section{Funding}

This research received no external funding. Conflict of Interests

The author declares no conflict of interest.

\section{ФАКТОРИ РИЗИКУ РОЗВИТКУ ЖІНОЧОГО БЕЗПЛІДДЯ НА ТРЕТИННОМУ РІВНІ НАДАННЯ МЕДИЧНОЇ ДОПОМОГИ У АКУРЕ, ПІВДЕННО-ЗАХІДНА НІГЕРІЯ}

T.A. Irinyenikan UNIVERSITY OF MEDICAL SCIENCES TEACHING HOSPITAL COMPLEX, AKURE, ONDO STATE, NIGERIA

Вступ. Неможливість пари народити спільних дітей є однією з головних психо-соціальних проблем, що призводить до дисгармонії подружніх стосунків.

Мета дослідження встановити можлиі фактори ризику розвитку жіночої неплідності.

Методи. У дослідження було залучено 400 жінок (200 - з неплідністю та 200 осіб склали групу контролю). Жінок було обрано методом випадкової вибірки у клініці планування сім'ї при Навчальнолікувальному комплексі Університету медичних наук, Акуре. Усі досліджувані заповнювали спеціально розроблений опитувальник. Усі випадки було класифіковано на первинне та вторинне непліддя. Застосовано статистичний регресійний аналіз: бінарну та непряму логістичну регресію, рівень значущості $p<0.05$.

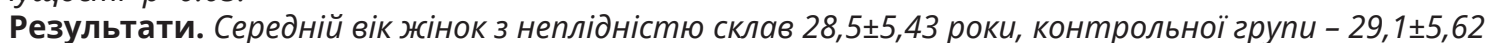
роки. У 155 (77,5\%) випадках мала місце вторинна неплідність, і лише у 45 (22,5\%) - первинне безпліддя. Статистично значущими факторами ризику були наявність фібротичних змін, оперативні втручання з їх приводу, кілька сексуальних партнерів, попередні аборти, синдром полікистозних яйників, хвороби, що передаються статевим шляхом та постабортний сепсис. 
Висновки. Дослідження показало, що вторинне безпліддя все ще переважає у структурі захворюваності, фактори ризику - мульфакторіальні. зусилля, спрямовані на зменшення розвитку непліддя через фактори, які можна попередити, повинні бути посилені.

KEY WORDS: жіноче безпліддя; фактори ризику; фіброз; синдром полікистозних яйників; хвороби, що передаються статевим шляхом.

\section{Information about the author}

Dr. Theresa Azonima Irinyenikan - Consultant Obstetrician and Gynaecologist, department of Obstetrics and Gynaecology, Faculty of Clinical Sciences, University of Medical Sciences/University of Medical Sciences Teaching Hospital Complex, Akure, Ondo State, Nigeria.

ORCID 0000-0002-5594-037X, e-mail: tirinyenikan2017@gmail.com

\section{References}

1. Vander BM, Wyns C. Fertility and Infertility definition and epidemiology. Clin Biochem.2018; 62:2-10.

2. Ombelet W. Global access to infertility care in developing countries: a case of human rights, equity, and social justice. FVV Obgyn 2012; Monograph: 7-16.

3. Mascarenhas $M N$, Cheung $H$, Mathers $C D$, Stevens GA. Measuring infertility in populations: constructing a standard definition for use with demographic and reproductive health surveys. Popul Health Metr 2012;10:1-11.

4. Adeyemi AS, Adekanle DA, Afolabi AF. Pattern of gynaecological consultations at Ladoke Akintola University of Technology Teaching Hospital. Niger J Clin Pract 2009;12:47-50.

5.Scarneciu I, Lupu S, Scarneciu C. Smoking as a risk factor for the development of erectile dysfunction and infertility in men; evaluation depending on the anxiety levels of these patients. Soc Behav Sci. $2014 ; 12$.

6. Olooto O, Wasiu E, Amballi A, Adebayo A, Banjo B, Taiwo A. A review of Female Infertility; important etiological factors and Management J. Microbiol. Biotech. Res., 2012,2(3):379-85.

7. Ombelet W, Devroey P, Gianaroli L, te Velde E (eds). Developing Countries and Infertility. Spec Issue Human Reprod 2008;1-117.

8. Nachtigall RD. International disparities in access to infertility services. Fertil Steril 2006;85:871-5.

9. Rutstein SO, Shah IH. Infecundity, infertility, and childlessness in developing countries. DHS Comparative Reports No. 9. World Health Organization, Geneva, Switzerland, 2004.

10. Sedgh G, Singh S, Shah IH, Ahman E, Henshaw SK, Bankole A. Induced abortion: incidence and trends worldwide from 1995 to 2008. Lancet 2012; 379:625-32.

11. Sule JO, Erigbali P, Eruom L. Prevalence of Infertility in Women in a South western Nigerian Community. African Journal of Biomedical Research 2008; 11: 225-7.

12. Orhue A, Aziken M. Experience with a comprehensive University hospital-based infertility program in Nigeria. Int J Gynaecol Obstet 2008; 101(1):11-15.
13. Abubakar AP, Yusuf TS. The profile of infertility in a Teaching Hospital in North West Nigeria. Sahel Medical Journal 2014;17: 1.

14. Okonofua FE. Infertility in Sub-Saharan Africa. In: Okonofua FE, Odunsi K, editors. Contemporary Obstetrics and Gynaecology for Developing Countries. Publishers: Women's Health and Action Research Center. Benin City: 2003:128-56.

15. Sabarre KA, Khan Z, Whitten AN, Remes O, Phillips KP. A qualitative study of Ottawa university students' awareness, knowledge and perceptions of infertility, infertility risk factors and assisted reproductive technologies (ART). Reprod Health J. 2013; 10(41):1-10.

doi:10.1186/1742-4755-10-41

16. Palihawadana TS, Wijesinghe PS, Seneviratne HR. Aetiology of infertility among females seeking treatment at a tertiary care hospital in Sri Lanka. Ceylon Med J. 2012; 57(2):79-83.

doi:10.4038/cmj.v57i2.4461

17. Orji EO. Comparative study of the impact of past pregnancy outcome on future fertility. Singapore Med J 2008; 49:1021-24.

18. Samiha M, Hassan AH, Nehad M, Fayek $E_{\text {, }}$ Gehan S. Risk Factors For Primary and Secondary Female Infertility in Alexandria: A Hospital Based Case Control Study. Journal of the Medical Research Institute JMRI 2006; 27(4):255-61.

19. Pastore LM, Karns LB, Ventura K, Clark ML. Longitudinal interviews of couples diagnosed with diminished ovarian reserve undergoing fragile $X$ permutation testing. J Genet Couns. 2014;23(1): 97-107.

doi:10.1007/s10897-013-9616-0

20. Tsai YH, Wang TW, Wei HJ, et al. Dietary intake, glucose metabolism and sex hormones in women with polycystic ovary syndrome (PCOS) compared with women with non- PCOS-related infertility. $\mathrm{Br}$ J Nutr. 2013;109(1):2190-8.

doi:10.1017/S0007114512004369

Received 13 September 2019; revised 11 October 2019; accepted 24 October 2019.

This is open access article distributed under the Creative Commons Attribution License, which permits unrestricted use, distribution, and reproduction in any medium, provided the original work is properly cited. 